\title{
DEFI Photonique: a French national training project for optics and photonics industry
}

\section{E. Boéri, E. Cormier}

E. Boéri, E. Cormier, "DEFI Photonique: a French national training project for optics and photonics industry," Proc. SPIE 9289, 12th Education and Training in Optics and Photonics Conference, 928923 (17 July 2014); doi:

$10.1117 / 12.2070706$

SPIE Event: 12th Education and Training in Optics and Photonics Conference, 2013, Porto, Portugal 


\title{
DEFI Photonique : a French national training project for optics and photonics industry
}

\author{
E. Boeri ${ }^{1,3^{*}}$, E.Cormier ${ }^{1,2}$ \\ ${ }^{1}$ PYLA platform, \\ ${ }^{2}$ Bordeaux University, \\ ${ }^{3}$ French Cluster « Route des Lasers » \\ Bordeaux, France
}

\begin{abstract}
The French government supports a structuring project for French Photonics. This project named DEFI Photonique is carried by the CNOP (National Committee for Optics \& Photonics) for a period of 5 years (20132017). One of the most important task is dedicated to training for industry, particularly SMEs. The project aims at elaborating a training offer based on the experience of PYLA, the Bordeaux training facility for Optics and Photonics, and create a national network throughout all the French Photonics clusters.
\end{abstract}

The project plans to initiate, develop and coordinate training courses based on the players skills in the sector, in particular regional clusters, depending on their field of excellence. This deployment of training courses should enable a mesh structure both thematically and geographically. Collaborative work between training players in each pole, including joint actions, will facilitate access to training courses for companies, especially SMEs.

A market survey is already being conducted in 2013 in photonics industry and application sectors. Implementation of actions involves all French photonics clusters as well as professional organizations.

We will rely on the feedback we have with PYLA to show how training courses can be a strategic tool for development of technologies and industries. At this stage of the DEFI Photonique project we will be able to present the results of different analyses that have been conducted in key sectors and plans that will be implemented for the realization of the first actions.

\section{KEYWORDS}

Continuing education, training courses for industry, network for continuing education

\section{CONTEXT}

CNOP (National Committee for Optics \& Photonics) brings together key French players in optics-photonics. Two players at the national level are AFOP (French association of optics and photonics industries) and SFO (the French Optics Society); At the regional level they include all the regional clusters: ALPhA Route des Lasers in Aquitaine, Photonics Bretagne, Elopsys in Limousin, Opticsvalley in Paris-lle de France, Optitec in the Riviera region, the Rhône Alpes optics cluster and Rhenaphotonics Alsace. Foundation of CNOP addressed the profession's need to be represented by a governing body. Its first action enabled stakeholders to get to know each other and to coordinate their actions. Today CNOP plays the role of spokesperson for the sector, addressing both the French Government and European instances. CNOP brought an early interest in the subject of education and training and established its first working group early 2004. When the CNOP decided to submit a proposal for the photonics sector structuration response to the French national call for

\footnotetext{
${ }_{2}^{1}$ www.pyla-routedeslasers.com

2 www.univ-bordeaux.fr

3 www.routedeslasers.com

* Corresponding author : e.boeri@pyla-routedeslasers.com
} 
projets to reinforce competitiveness in small and medium size photonic industries in strategic industrial fields and sectors, it has included training in its project as a major focus for the industry.

The DEFI Photonique project, accepted and funded by the French authorities, does span across all areas of the French optics and photonics industrial sector.

DEFI Photonique aims to satisfy two fundamental needs:

- To define the industrial strategy and to structure the photonics sector in order to improve enterprise competitiveness and to accelerate the development of SMEs in the photonics industry.

- To offer services and actions open to all companies on the French national territory, and especially to help very small to medium sized enterprises to define/improve their industrial strategy, to facilitate their entry/penetration of photonics applications markets and to anticipate their needs and their clients's needs in terms of competencies.

DEFI Photonique, sponsored by the Sagem and Thales groups, will proceed along two main lines of development and offer services or growth accelerators for the photonics industrial sector.

- Axe 1: To offer specific accompaniment to small and medium sized photonics enterprises in chosen fields dedicated to promote growth and development.

- Axe 2: To offer a continuous training program adapted to meet the strategic stakes \& objectives of the photonics industry.

\section{PROJECT DESCRIPTION}

The emergence of new technologies and especially the increasing diversity of photonics applications raise the question of adapting human resources to new know-how and markets. Skills shortages and a lack of qualified personnel is a barrier to the development of this innovative sector in all fields from technology transfer to production and use

The needs cover the entire value chain and skill levels. Moreover, the wide variety of applications due to the enabling technology characteristic of photonics, put the training task at the interface of several disciplines which increases the difficulty of companies, particularly SMEs, facing human resource issues.

The French photonics environment has a strong high-level academic education system with its engineering schools and universities, but its existing offer of continuing training courses is scarce, dispersed and not always meets very specific technical and short term needs of industry. Continuing education is necessary not only for SMEs in the field but also in the application sectors (medical, aerospace, mechanical ...) to accompany the penetration and development of technologies and photonic systems in these areas.

The aim of the action is:

- initiate, develop and coordinate a network of continuing education based on the skills of players in the sector, in particular regional clusters, depending on their areas of excellence. This deployment of training courses should enable a mesh both thematically and geographically;

- $\quad$ promote training practices among industry, encourage the expression of needs for continuing training and offer a single window for people who want to undergo continuing training in optics and photonics;

- accompany the development of technologies in training users in applications of photonics and facilitate the close matching of technological developments with skills evolution;

The added value of the action is in extending the supply of continuing training at the national level and involvement in the design of new training formats. We intend to build a system of training courses covering the entire value chain to provide companies an adapted response to their development needs of skills and tools to the challenge efficiently the SME market.

This new national platform will rely on the concept of PYLA platform that will pilot the whole action.

Created in 2005 by the Université Bordeaux 1, the Université Bordeaux 2, CEA-Cesta and Alpha-Route des Lasers, PYLA is a vocational training platform with first-rate educational/technological facilities.

PYLA gathers together and shares the skills of the industry's various players and collaborates with recognized training and research organizations, so as to offer a catalogue of over 40 training courses. The courses call upon over 70 instructors, all experts in their respective fields. 
They are intended for a broad public of photonic-technology designers, manufacturers, integrators and users, no matter their level of qualification.

PYLA also designs and carries out tailor-made training courses, suited to the needs of SMEs, major groups and research institutes, at both the national and European levels.

Pyla will make available its expertise in short course design, organization of training courses and practical training, administrative management, communication and marketing of training offers. It will expand its activity across the country in collaboration with all regional clusters.

The project will also prepare training solutions for the future, including being involved in the development of elearning.

It will also lead a discussion on the recognition of training mainly through a system of certification of programs, trainers and participants especially in the newly regulated field of optical safety.

\section{PROJECT ORGANIZATION AND MILESTONES}

Organization and management

For this axis of $D E F I$ Photonique, a specific steering committee was established early in the project to monitor and guide the actions. It consists of one representative from each member of the CNOP to take into account the interests of the sector at national level.

\section{Market Survey}

A special working group was set up to participate in the first step of market survey. The first difficulty is to define the themes and application areas on which to focus the study as it is quite impossible to cover all applications of photonics. The Working Group has considered the recommendations of the CNOP from 2010 report, which defines 21 technological orientations, covering 6 fields, emerging as priorities, in the sense that France has real advantages for its scientific and industrial development. We also took into account the state of the training offer across sectors. The selected thematic areas are: telecommunications, health and living systems, display and augmented reality, industrial processes and optical measurements, sources, sensors and sensor networks, lasers, new materials and components.

The study will consist of an interview of companies involved in photonics, covering selected areas. The working group has designed a draft of the interview guide. The aim is that companies talk openly about their business and strategy, including the role that can have the training to support the development of their activity, the type of the client and the role that can have the training of their client in the development of their market. More specifically, we need to talk about how they train their employees, how they fund these training periods, which are the potential barriers to use of training ... Legal, social and environmental constraints are also taken into account. Companies may also be involved in the development of a training offer by providing to disseminate their expertise either for their customers, for the entire community. We must also identify this potential to be integrated into the construction of training programs that we can offer.

At the same time, the working group has formed a panel of about 140 companies among members of their clusters and covering selected sectors. It will make a first interview with the company in order to qualify the contact and ensure that the company wants to participate in the study. Detailed interviews will then be conducted by a consultant in market research. We target to interview at least 70 companies.

The interview guide will first be finalized with the consultant before starting the interviews and then it will eventually be finely retuned after the first series of five to ten interviews. The panel may also change depending on the answers we discover to focus on a thematic field over another or to investigate directly among end users.

Throughout the investigation, the Working Group regularly monitors the progress and look at the contents of the interviews. 
A first level of analysis and recommendations will be provided by the consultant. Based on the results, we can continue the investigations on specific topics by organizing thematic workshops with companies and stakeholders involved in the training.

\section{Analysis of the existing supply, identification of resources and positioning.}

All existing training offer must be identified and listed. In the heart of photonics, it is provided by the major players in education and training as PYLA or the Institut d'Optique Graduate School. Some applicative areas are also covered when the volume and size of firms in the market are substantial (e.g. lighting or the use of fiber optics in telecommunications). This work will allow us to identify shortcomings but also to identify existing training courses that could be marketed to companies as part of our action.

At the same time, we need to identify all the expertise and resources that could be mobilized for the implementation of training. Expertise should be sought in training organizations, schools, universities, laboratories but also in the companies themselves. Technical resources are also available in these places and more often in the form of open through public-private partnerships for research and development platforms. $D E F I$ Photonique can give the opportunity to organizations to promote their skills, expertise and technical resources through continuing education.

For each resource, it is necessary to study the nature of partnerships that can be established. Depending on the nature of the contributor (technical expertise or resources) will face administrative, economic, competitive, legal constraints.

When mapping skills and resources will be completed as well as various technical opportunities to build training programs are identified, the steering committee will be responsible to make choices and recommendations. It is important that each cluster can position and support training activities on its own territory according to its strategic areas. Overall, the actions of each territory should be complementary while respecting geographic coverage of training activities, in response to business needs.

\section{CONCLUSION}

Market research and positioning study are both underway. We already have some results from interviews that have started in recent weeks. This study phases will be completed by November 2013 and available analyzes will allow us to develop a strategy for development of training courses to meet the needs of the industry.

The involvement of different players in education and training, the use of technical resources available in the entire country and forged partnerships enable the creation of a network for continuous training in photonics in France. The animation of this network will provide a single portal for companies.

In total over the period 2013-2017, we plan to introduce and implement more than twenty new training courses which can then be sustained through the whole network. 